\title{
Assessment of a two-way coupling methodology between a flow and a high-order nonlinear acoustic unstructured solvers
}

\author{
Adrien Langenais • François Vuillot • Christophe Peyret • \\ Gilles Chaineray · Christophe Bailly
}

\begin{abstract}
A two-way coupling on unstructured meshes between a flow and a high-order acoustic solvers for jet noise prediction is considered. The flow simulation aims at generating acoustic sources in the near field while the acoustic simulation solves the full Euler equations, thanks to a discontinuous Galerkin method, in order to take into account nonlinear acoustic propagation effects. This methodology is firstly validated on academic cases involving nonlinear sound propagation, shock waves and convection of aerodynamic perturbations. The results are compared to analytical solutions and direct computations. A good behaviour of the coupling is found regarding the targeted space applications. An application on a launch pad model is then simulated to demonstrate the robustness and reliability of the present approach.
\end{abstract}

Keywords Two-way Navier-Stokes-Euler coupling · Nonlinear acoustics · High-order solver · Unstructured grids

\section{Introduction}

High speed jet noise is a major issue in aeronautics and astronautics which has been extensively investigated as reviewed by Seiner [1], Tam [2] or Bailly and Fujii [3] for instance. Imperfectly expanded hot supersonic jets generated by rocket motors at lift-off induce severe acoustic loads on the launcher and its payload [4] as illustrated in figure 1. The main contributions include the noise generated by the free part of the jet, the impinging region inside the flame trench, the noise emitted at the end of the flame duct and the reflected and diffracted noise on the launch pad. In addition to various experimental studies [5] which have led to significant noise reduction on launch pads [6], semi-empirical jet noise models based on the Lighthill's theory have been formulated [7] and improved [8-10] but they are often unsuitable to deal with such realistic space applications. More recently, the development of large-eddy simulation (LES) allowed accurate simulations of hot supersonic jets [11-15], even for increasingly complex configurations as performed with realistic launch pad geometries by Fujii et al. [16] or Tsutsumi et al. [17]. The computational cost of such simulations remains however heavy for current industrial practices, especially when the acoustic propagation is directly calculated by the flow solver. The cost may be reduced by considering an integral formulation to extrapolate the acoustic far field from a control surface $[18,19]$. This technique implemented in the KIM code [20] has been employed at ONERA in past and recent works for supersonic jet noise applications $[13,21]$. It is usually efficient but installation effects induced by the geometry and nonlinear propagation effects induced by the high acoustic levels for supersonic flows [22] are not readily taken into account.

One-way coupling methods between a flow solver and an aeroacoustic solver based on Euler equations are more and more implemented to take care of possible mean flow effects. Various strategies in terms of meshing and coupling algorithms have already been assessed [23,24]. The Navier-Stokes equations (NS)

A. Langenais $*$ F. Vuillot $\cdot$ C. Peyret · G. Chaineray

ONERA, DMPE, University of Paris-Saclay, F-91123 Palaiseau, France

E-mail: adrien.langenais@onera.fr

C. Bailly

LMFA UMR 5509, École Centrale de Lyon, University of Lyon, F-69134 Écully, France

E-mail: christophe.bailly@ec-lyon.fr 


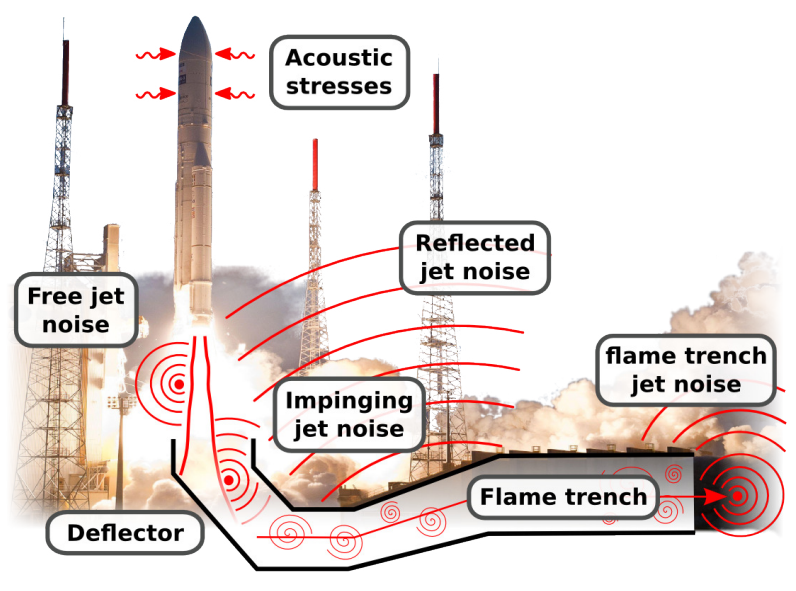

Fig. 1: illustration of the acoustic ambiance at launcher lift-off.

have been firstly weakly coupled to the linearized Euler equations (LEE) as presented in the works of Djambozov et al. [25] or Bogey et al. [26]. More recently, one-way couplings between NS equations and full Euler equations have been performed as reported by Sescu et al. [27] (2D subsonic and supersonic jet cases with structured solvers), Harris et al. [28] (2D application to the SLS acoustic ambiance with unstructured solvers) or Labbé et al. [29] (3D subsonic jet case with structured LES and unstructured Euler solver). In particular, Tsutsumi et al. [17] have simulated an impinging jet inside various flame trench geometries thanks to a structured flow solver. The radiated waves are propagated in the far field with a Euler solver to take into account reflection, diffraction and nonlinear effects. The Mach 3.3 jet simulated by de Cacqueray and Bogey [22] with a structured flow solver can also be mentioned. The noise is propagated both via a LEE solver and a full Euler solver, leading to an overall $5 \mathrm{~dB}$ reduction around the peak radiation direction at 240 radii from the source, when the nonlinear effects are taken into account. One-way coupling methods are however unsuitable for the simulation of realistic installation effects and possible acoustic feedback on the flow while these phenomena can have a significant impact on the solution. For example, it can be seen in a coupled computation from Tsutsumi et al. [17] that strong waves radiated up to the nozzle exit area. It suggests that two-way coupling methods need also to be investigated. Implementations of such methods can be found in the works of Borrel et al. [30] or Léger et al. [31] for two-dimensional and Labbé et al. [32] for tri-dimensional applications with structured solvers, but tri-dimensional fully unstructured configurations are quite rare. Langenais et al. [15] have carried out a two-way coupled unstructured NSEuler computation to simulate the noise generated by an overexpanded Mach 3.1 free hot jet. Encouraging results have been obtained since nonlinear effects have been highlighted and the methodology has led to a significant predictivity improvement. However, the free jet case does not involve any major feedback or installation effect. The ability of the implemented two-way coupling methodology to deal with such effects has to be assessed before being applied to more realistic space configurations.

Important efforts have been initiated to establish a reference benchmark data-base for computational aeroacoustic (CAA) problems. The main contribution has been provided by the NASA workshops [33-36]. Numerous problems and associated analytical solutions are proposed, to test the effectiveness of boundary conditions [33,36], acoustic diffraction by obstacles [34,36], propagation in ducts [34], propagation in transonic and supersonic nozzles $[33,35]$ and acoustic generation and refraction in shear layers [35, 36] for example. Benchmark problems have then been applied to various numerical methods for validation purposes. In particular, Harris et al. [28] have applied a one-way NS-Euler coupling method to a 2D twocylinder acoustic scattering problem, Léger et al. [31] have also validated a two-way LEE-LEE coupling method on several 2D problems of diffraction by cylinders and finally, Labbé et al. [29] have simulated a 2D convected isentropic vortex, as proposed first by Yee et al. [37], using a one-way NS-Euler coupling method. However, the ability of a two-way coupling method to handle nonlinear propagation effects, acoustic feedback effects and realistic installation effects without significant spurious noise is not often assessed in the literature.

In the present article, a tri-dimensional two-way NS-Euler coupling methodology is presented and validated on cases of increasing complexity. Computational grids are unstructured in both flow and acoustic 
solvers. The full Euler equations are solved in the acoustic code via a high-order discontinuous Galerkin method. This hybrid approach is thus able to deal with acoustic feedback on flow, nonlinear propagation effects and complex three-dimensional geometries as required for realistic space applications. The manuscript is organized as follows. Computational tools are introduced in section 2 with particular focuses on the coupling procedure in subsection 2.3 and the benefit of the use of high spatial orders in subsection 2.4. The coupling methodology is applied to several academic cases in section 3: a pulsating sphere in subsection 3.1, a wave guide in subsection 3.2, a standing wave in a cavity in subsection 3.4, a shock tube in subsection 3.5 and a convected isentropic vortex in subsection 3.6. Finally, an application on a launch pad model is performed in section 4 and concluding remarks are provided in section 5 .

\section{Computational tools}

\subsection{Flow simulation}

In previous studies on supersonic jet noise $[13,15,21]$, the simulation of the turbulent flow and its near field acoustics was achieved with the multi-physic and multi-species ONERA code CEDRE [38] used here as the flow solver. The Navier-Stokes equations (NS) are solved by a cell-centered finite volume method on generalized unstructured grids. Species viscosity is calculated with the Sutherland law. The spatial discretization is performed with a second-order MUSCL method and an HLLC flux scheme. In the following computations, meshes are here entirely made of tetrahedral elements. The time integration is performed with a second-order explicit or implicit Runge-Kutta scheme. A constant time step is set in order to respect the acoustic criterion

$$
\mathrm{CFL}=\left(\bar{u}+c_{0}\right) \Delta t / \Delta x<1
$$

in the acoustic near field, where $\bar{u}$ is the mean flow velocity, $c_{0}=\sqrt{\gamma r T}$ the speed of sound, $\Delta t$ the time step and $\Delta x$ the characteristic size of a tetrahedron. The latter is chosen equal to the diameter $d$ of a regular tetrahedron inscribed sphere, namely $d=a \sqrt{6} / 6$ where $a$ is the tetrahedron edge length set for the unstructured mesh generation. The resulting maximum value in the whole domain is typically $\mathrm{CFL} \leqslant 0.85$ in all cases which observes the acoustic criterion. The spatial resolution is quantified in terms of points per wavelength (PPW) for the considered meshes, defined as

$$
\mathrm{PPW}=\frac{c_{0}}{f \times \Delta x}
$$

where $f$ is the frequency and $\Delta x=d$. Preliminary computations have allowed to establish that a spatial resolution of $\mathrm{PPW}=40$ is required to get a numerical damping less than $0.1 \mathrm{~dB} / \lambda$, where $\lambda=c_{0} / f$, for a given frequency at the $2^{\text {nd }}$ order in time and space. This resolution is twice as usually considered for jet noise application with the CEDRE code [13]. Note that in significant grid stretching region, the provided resolution calculation is always based on the largest cell.

\subsection{Computational aeroacoustics}

The nonlinear Euler equations are solved with a nodal discontinuous Galerkin method (DG) on unstructured grids implemented in the ONERA code SPACE [31,39]. This method combines characteristics from both finite elements and finite volumes methods: the solution in an element is approached with a polynomial representation which is discontinuous between elements while numerical fluxes are exchanged at the element faces. High spatial orders can be easily implemented within this formulation. In the present work, second to fourth-order elements are used (see §2.4). The time integration is performed with a second-order explicit Runge-Kutta scheme. The required spatial resolution has been succinctly assessed in Delorme et al. [39] for ideal unstructured grids and turned out to be PPW $=14$ at second-order and PPW $=3$ at fourth-order. The PPW metric is defined as previously in equation (2) and always based on the largest cell in a region. Preliminary computations [40] have also shown that these criteria result in a numerical dissipation in the order of the molecular viscous damping, i.e. $\sim 10^{-3} \mathrm{~dB} / \lambda$. The external non-reflective boundary condition is provided by a formulation based on the characteristic theory [41] which allows the outcoming acoustic fluctuations $\left(p^{\prime}, T^{\prime}, \boldsymbol{u}^{\prime}\right)$ around the imposed mean flow $\left(p_{\infty}, T_{\infty}, \boldsymbol{u}_{\infty}\right)$ to be efficiently damped. A shock-capturing scheme based on a velocity divergence sensor [42] is also implemented in SPACE. Finally, thermodynamic properties of the propagation medium (air) are constant with $\gamma=1.4$. 
The standalone SPACE code has already been carefully numerically assessed in terms of dispersion, dissipation and convergence error [31], and also applied to academic validation cases such as a convected isentropic vortex [29], diffraction by one or multiple circular obstacles [31], duct acoustic propagation [43] and wave propagation in a sheared flow [32].

\subsection{Coupling procedure}

The link between the two solvers CEDRE and SPACE is a two-way surfacic coupling without using a mesh overlapping contrary to what is often implemented in existing approaches [23, 27,30,31]. Boundary point location and data exchange are operated via MPI communications thanks to the open source CWIPI coupling library proposed by Quemerais from ONERA $[38,44]$. The same space discretization is considered at the interface since conformal tetrahedral meshes are here used. The coupling algorithm is described in detail in figure 2. It consists in locating boundary cells on each side of the surfacic interface, then calculating and exchanging values required by the other code at cell centers for CEDRE and nodes for SPACE, every time step. The constant time step is the same in NS and Euler regions. The identical space and time discretization ensures that the signal degradation which could be induced by spatial or temporal aliasing [45] is minimized. Since conservative values sent to SPACE are calculated from CEDRE primitive values $(p, T, \boldsymbol{u})$ with SPACE thermodynamic laws $(\gamma=1.4)$, multi-species NS simulations can be carried out without pressure, temperature or velocity discontinuity across the interface. Moreover, the procedure does not require the implementation of brand new coupling boundary conditions in each code. Ghost cells of an existing condition in CEDRE are indeed emulated and fed with incoming SPACE values. In the same way, the conservative variables $(\rho, \rho \boldsymbol{u}, \rho e)$ at the SPACE boundary are reassessed with incoming CEDRE boundary values. At the end of a coupling cycle, exchanged values are used by each of the solvers to compute its numerical fluxes at the interface where the conformal grids ensure the conservation laws. This algorithm only requires boundary values and the data exchanges are fully managed by the coupling library, which might be easily generalized to other solvers.

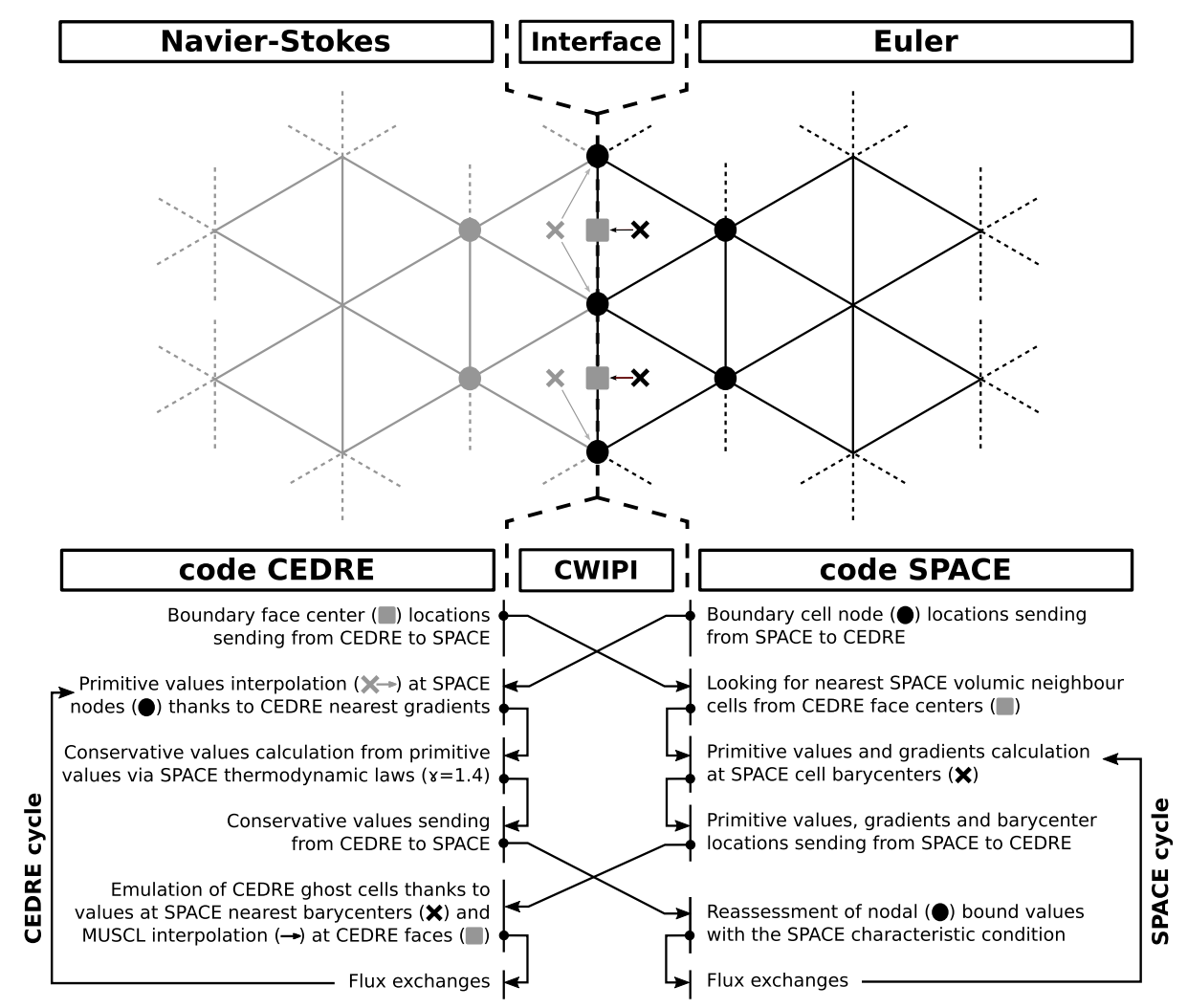

Fig. 2: diagram of the NS-Euler two-way coupling procedure performed at every time step. 
The coupling interface is typically placed in a turbulence-free zone. Since the NS-Euler methodology does not imply any constraint on secondary flows or acoustic levels, it can be brought very close to the sources generated by the primary flow. Consequently, vortices can intermittently cross the interface. However, no viscous effect is expected to occur except for wall bounded flows.

\subsection{High-order scheme and order mapping}

The need of highly accurate numerical methods in computational aeroacoustics to propagate waves in the far field with low dissipation and dispersion is now well recognized [46]. High spatial orders implemented in SPACE allow accurate propagation with less than PPW $=5$ and consequently require meshes with fewer cells. It has been checked that using fourth-order elements instead of second-order elements while decreasing the number of cells reduces the calculation cost for the same given accuracy. High-order discontinuous Galerkin methods remain costlier than finite differences or integral methods but they are presently more convenient for realistic applications thanks to the Euler solver and the unstructured grid implemented in SPACE. Furthermore, the second-order Runge-Kutta scheme does not reduce the global simulation quality since the NS computation is also second-order accurate. Validation cases presented in next section are performed with second-order elements. Nevertheless, a non regression using the third and fourth spatial orders has been successfully controlled on each of them.

The coupling procedure uses conformal tetrahedral meshes at the interface to avoid the spatial aliasing and to ensure the robustness. Consequently, the methodology has to deal with both the disparity of scheme order between the NS and the Euler regions, and a significant grid stretching near the coupling interface in the Euler region, which induces an accuracy discontinuity and can lead to spurious numerical oscillations [31]. An order mapping method (also called $h p$-adaptation) is used in the Euler region associated with the DG solver [43]. In order to illustrate the locally adapting element order, a part of the acoustic mesh further introduced in section 4 is shown in figure 3. A layer of second-order elements nearby the interface avoids the accuracy discontinuity with the NS domain and the order is adapted everywhere else according to the cell size. In particular, the order is lower where geometric details force mesh refinement in order to remain consistent in accuracy in the whole computational domain. It has been shown $[31,43]$ that the order mapping enables a better compromise between numerical cost, accuracy and robustness.

\section{Validation}

The two-way coupling methodology is first validated on two and three-dimensional academic cases. Propagation of spherical and planar acoustic waves is computed in linear and nonlinear regimes in order to assess the one-way (NS $\rightarrow$ Euler) acoustic coupling. A planar standing wave is further simulated in a cavity in order to assess the two-way acoustic coupling. A shock tube is also considered. For all planar wave cases,

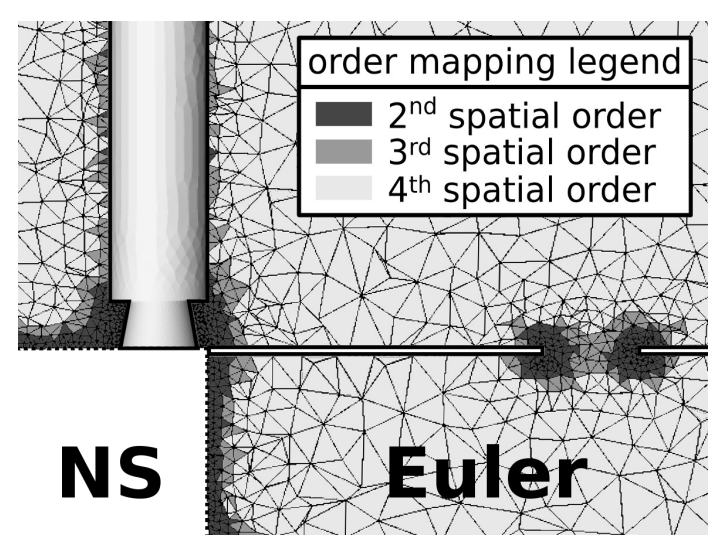

Fig. 3: Euler computational domain (CAA) using the order mapping method. interface. 
the coupling interface is tilted at $0^{\circ}$ or $33^{\circ}$ regarding the propagation direction to quantify the spurious noise induced by non-normality. Finally, the convection of a $2 \mathrm{D}$ isentropic vortex is simulated in order to assess the ability of the two-way coupling to handle the crossing of vortical structures at the interface without emitting too much spurious noise. Hereinafter, superscript ${ }^{*}$ is associated with coordinates or lengths made dimensionless by the acoustic source wavelength $\lambda_{s}$, subscript $s$ with the acoustic source and int with the interface. The distance from the source in the propagation direction is denoted $z$. Numerical domains are always composed of NS and Euler sub-domains connected by the coupling interface at location $z_{\text {int }}^{*}$.

\subsection{Pulsating sphere}

The pulsating sphere is modelled as a source of mass located at the NS sub-domain center, generating spherical waves thanks to sinusoidal source oscillations at the frequency $f_{s}=250 \mathrm{~Hz}$ corresponding to a wavelength $\lambda_{s}=1.39 \mathrm{~m}$. This acoustic source is compact since $r_{s}^{*}=r_{s} / \lambda_{s}=1 / 20$ with $r_{s}$ the source radius. Low and high acoustic levels are considered with a SPL $=114 \mathrm{~dB}$ and a SPL $=174 \mathrm{~dB}$ at $z^{*}=r_{s}^{*}$. The spherical NS sub-domain defined by $r_{s}^{*}<z^{*}<z_{i n t}^{*}$ is surrounded by a larger Euler sub-domain defined by $z_{i n t}^{*}<z^{*}<7.2$ where $z_{i n t}^{*}=0.72$, as shown in figure 4 . The mesh refinement corresponds to a cut-off frequency of $f_{c}=600 \mathrm{~Hz}$ which is high enough to properly discretize the first harmonic $2 f_{s}=500 \mathrm{~Hz}$. The propagation medium is air at rest with $T_{0}=300 \mathrm{~K}$ and $p_{0}=101325 \mathrm{~Pa}$.

The NS-Euler coupling provides good results in the linear regime. The measured speed of sound $c=$ $347.3 \pm 0.1 \mathrm{~m} / \mathrm{s}$ is nearly equal to the theoretical value $c_{0}=\sqrt{\gamma r T}=347.2 \mathrm{~m} / \mathrm{s}$ and the classical $1 / r$ spherical decay is found in figure 5(a). The numerical dissipation is estimated by comparing the wave amplitude with the theoretical value for inviscid fluids. The dissipation remains close to the expected molecular damping of about $10^{-3} \mathrm{~dB}$ per wavelength [47]. The present coupling results are also compared with those obtained with a direct noise computation provided by NS equations and a wave extrapolation method based on a control surface, namely the Ffowcs-Williams \& Hawkings integral method (FWH) implemented in the KIM code [20]. Given the $1 / r$ amplitude decay for spherical waves, nonlinearities are found insignificant even at high level. Consequently, the coupling and the direct method agree well with the linear analytical solution as displayed in figure 5(b). The pulsating sphere case validates the coupling behaviour in the linear propagation regime, but does not enable to properly study nonlinear effects because of the geometrical expansion.

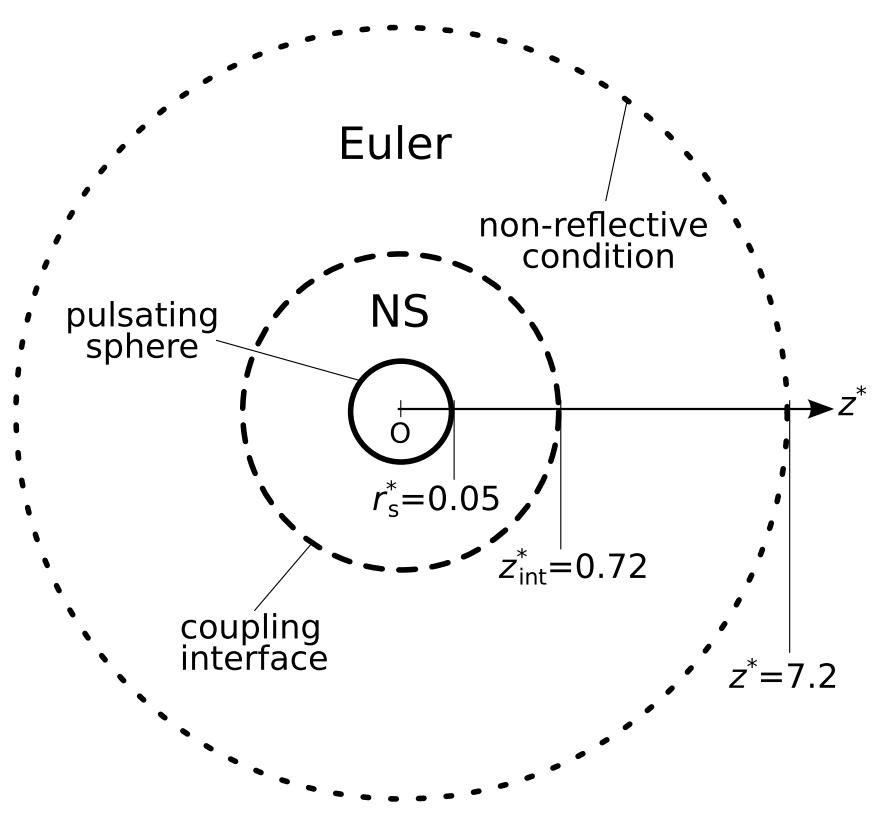

Fig. 4: sketch of the computational domain for the pulsating sphere. 


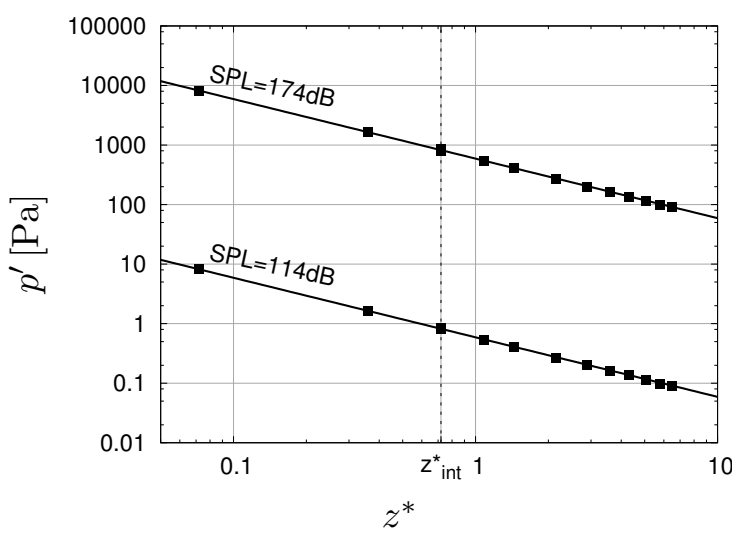

(a)

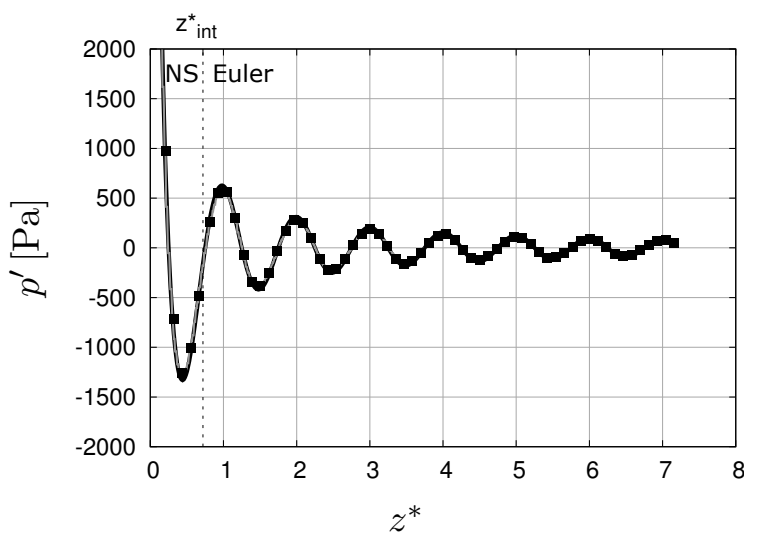

(b)

Fig. 5: (a) amplitude decay and (b) radial profile of spherical waves.

: direct simulation; $\mathbf{a}:$ NS-Euler coupling.

\subsection{Wave guide}

In the wave guide case, velocity fluctuations are imposed at one end $\left(z^{*}=0\right)$ of a guide with dimensions $(0.72 \times 0.72 \times 14.4) \lambda_{s}^{3}$ as shown in figure 6 . The generated planar waves propagate through the NS subdomain defined by $0<z^{*}<z_{\text {int }}^{*}$ and in the Euler sub-domain defined by $z_{\text {int }}^{*}<z^{*}<7.2$. A buffer zone aims at damping waves before they reach the non-reflective boundary condition at $z^{*}=14.4$ thanks to gradually stretched cells. The source frequency, the mesh cut-off frequency and the ambient medium properties are identical to the previous case.

For a low amplitude, similar features in terms of propagation velocity and dissipation are observed for the pulsating sphere and the wave guide cases. However, the latter shows strong nonlinear propagation effects at higher level. Each compression phase tends to stiffen up to a weak shock formation as drawn in figure 7. A nonlinear analytical solution based on the Blackstock model [48] can be considered for validation. It consists in connecting the Fubini solution to the Fay solution, the first one (respectively the second) being only valid before (respectively after) the weak shock formation due to nonlinear propagation. This analytical solution can be expressed for the pressure fluctuation in the time domain at a distance $z^{*}$ from the source as a sum of harmonics,

$$
p^{\prime}\left(z^{*}, t\right)=p_{s} \times \sum_{n=1}^{\infty} B_{n} \sin \left[n\left(\omega_{s} t-2 \pi z^{*}\right)\right]
$$

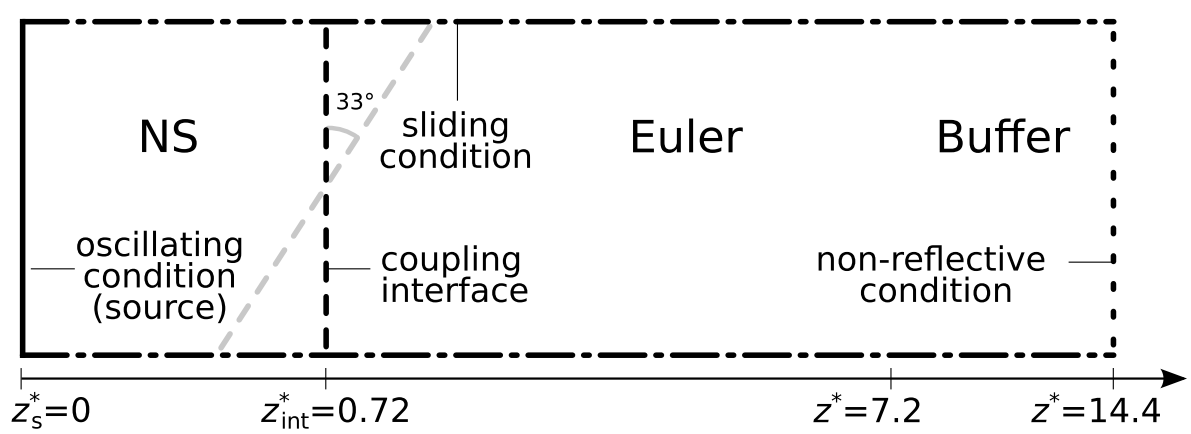

Fig. 6: sketch of the computational domain for the wave guide. 
where the coefficients $B_{n}$ are given by

$$
B_{n}=\frac{2 \overline{z^{*}}}{n z^{*}} J_{n}\left(n \frac{z^{*}}{\overline{z^{*}}}\right)
$$

for the Fubini solution and by

$$
B_{n}=\frac{2 \overline{z^{*}}}{n\left(\overline{z^{*}}+z^{*}\right)}
$$

for the Fay solution. In these expressions, $p_{s}$ is the source amplitude, $n$ the harmonic order, $\omega_{s}$ the source pulsation and $J_{n}$ the first kind Bessel function of order $n$. The distance $\overline{z^{*}}=\left(\rho_{0} c_{0}^{2}\right) /\left(2 \pi \beta p_{s}\right)$ is the shock formation length without viscous effects [48] where $\rho_{0}$ and $c_{0}$ are the density and the speed of sound in the ambient medium, and $\beta=(\gamma+1) / 2$ the nonlinearity coefficient for a perfect gas. A good agreement between this analytical solution, the direct simulation and the NS-Euler coupling is found for the $\mathrm{SPL}=174 \mathrm{~dB}$ as shown in figure 7 despite slight overshoots in the vicinity of the shocks for both numerical solutions.

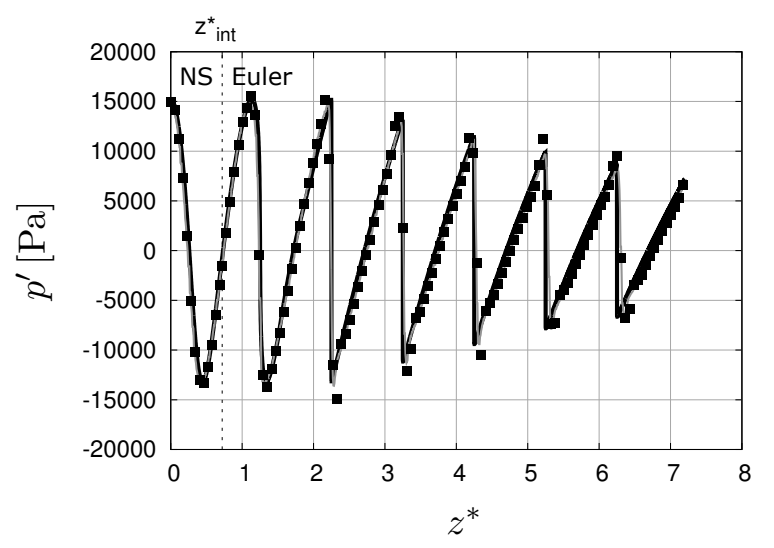

Fig. 7: planar wave distortion due to nonlinear propagation at SPL $=174 \mathrm{~dB}$. —: analytical solution [48]; - direct simulation; $\mathbf{\square}$ : NS-Euler coupling.

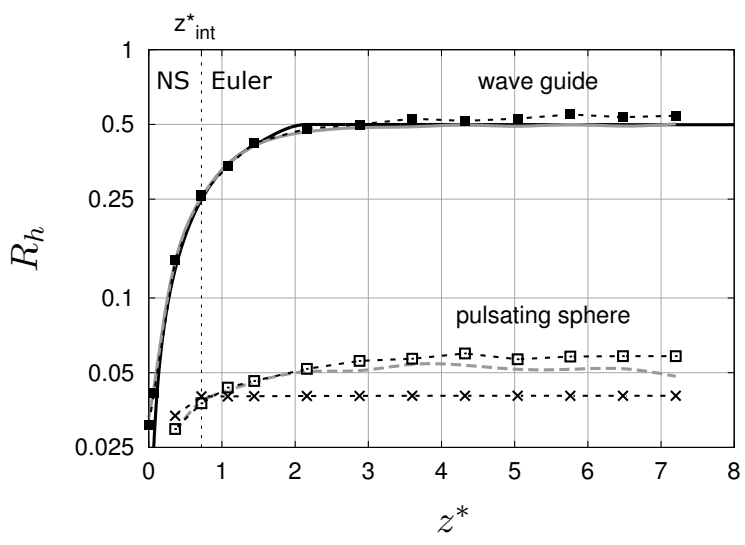

Fig. 8: harmonics generation measured with the $R_{h}$ ratio in log scale for the SPL $=174 \mathrm{~dB}$. Pulsating sphere case: $=-=$ : direct simulation; --×--: NS-FWH; -- --: NS-Euler coupling. Wave guide case: - analytical solution [48]; $\longrightarrow$ : direct simulation; --a- - : NS-Euler coupling. 


\subsection{Frequency analysis}

A metric proposed by Gallagher and McLaughlin to quantify acoustic nonlinearities [49] is introduced to compare the numerical results provided by different methods. The harmonic ratio $R_{h}$ is defined as the ratio of the Fourier transform magnitude of the first harmonic $(500 \mathrm{~Hz})$ to the fundamental one $(250 \mathrm{~Hz})$. Because nonlinear waves distortion is equivalent in the spectral domain to an energy transfer from low to high frequencies, also called spectral spreading, $R_{h}$ increases when nonlinear effects occur as characterized in figure 8 for both the pulsating sphere and the wave guide cases. In the pulsating sphere case, note that Ffowcs-Williams \& Hawkings (FWH) results provide a flat curve corresponding to a linear wave extrapolation, as expected for such an approach. For this case, the coupling and the direct simulation show similar trends despite that nonlinear effects are weak and that the measurement dynamic remains limited. On the contrary in the wave guide case, nonlinear effects are significantly stronger as highlighted previously and a higher dynamic is obtained. The $R_{h}$ ratio quickly increases up to 0.5 which is the theoretical asymptotic value when the wave shape turns to sawtooth patterns. Harmonics have already received a large amount of energy when the wave reaches the coupling interface. Analytically, $R_{h}$ is given by

$$
R_{h}=\frac{B_{2}}{B_{1}}
$$

from equations (4) and (5) with $n \in[1,2]$. A particularly good agreement is found between the analytical solution, the direct computation and the coupling method which validates the latter for nonlinear acoustic propagation.

For completeness purposes, the energy conservation across the coupling interface is checked using the wave guide configuration. Fourier transforms are performed on each side of the interface to quantify acoustic energy variations. Both acoustic levels $114 \mathrm{~dB}$ and $174 \mathrm{~dB}$ are studied, with and without a mean flow at a Mach number of $M=u / c=0.2$, with and without a tilted interface. The cut-off frequency of the mesh is $f_{c}=600 \mathrm{~Hz}$. Results are reported in table 1 . Spurious noise is generated at the interface crossing depending on the tested conditions. On the other hand, the energy of the fundamental frequency $\left(f_{0}=250 \mathrm{~Hz}\right)$ is always well transmitted from NS to Euler regions since the maximum variation just reaches $1 \%$ for the most unfavourable case. Greater discrepancies of 2 to $4 \%$ are found concerning the first harmonic near the cut-off frequency. Beyond the cut-off frequency, noise introduction remains moderate knowing the possible aliasing effect and that higher harmonic magnitudes are small compared to that of the fundamental in the Fourier transform. Regarding the influence of the input parameters, the initial acoustic level seems to have a limited impact on the overall spurious noise generation, as the mean flow. In particular, the noise does not increase with. On the contrary, the interface tilt angle has a significant impact, showing that non normality is the primary source of spurious noise. Last column highlights that variations of the overall energy, i.e. integrated over the whole spectrum, are in the order of that of the

\begin{tabular}{cccccccc}
\hline \multirow{2}{*}{$\mathrm{SPL}$} & \multirow{2}{*}{ tilt } & Mach & \multicolumn{3}{c}{ discrete frequencies } & \multicolumn{2}{c}{ broadband } \\
\cline { 4 - 8 } & & & $250 \mathrm{~Hz}$ & $500 \mathrm{~Hz}$ & $750 \mathrm{~Hz}$ & $\sum_{n}$ & $\sum_{n}$ \\
& & $n=1$ & $n=2$ & $n=3$ & $n>1$ & $0 \leq n \leq 20$ \\
\hline \multirow{2}{*}{$114 \mathrm{~dB}$} & $0^{\circ}$ & 0 & $0.22 \%$ & - & - & - & $0.22 \%$ \\
& & 0.2 & $0.12 \%$ & - & - & - & $0.12 \%$ \\
$114 \mathrm{~dB}$ & $33^{\circ}$ & 0 & $1.06 \%$ & - & - & - & $1.06 \%$ \\
& & 0.2 & $0.65 \%$ & - & - & - & $0.65 \%$ \\
$174 \mathrm{~dB}$ & $0^{\circ}$ & 0 & $0.12 \%$ & $2.11 \%$ & $4.54 \%$ & $2.57 \%$ & $0.30 \%$ \\
& & 0.2 & $0.22 \%$ & $2.19 \%$ & $3.17 \%$ & $2.29 \%$ & $0.28 \%$ \\
$174 \mathrm{~dB}$ & $333^{\circ}$ & 0 & $1.02 \%$ & $3.87 \%$ & $9.07 \%$ & $5.09 \%$ & $1.32 \%$ \\
& & 0.2 & $0.64 \%$ & $2.58 \%$ & $6.04 \%$ & $2.83 \%$ & $0.70 \%$ \\
\hline
\end{tabular}

Table 1: spurious energy generation rate of planar acoustic waves when crossing the coupling interface from NS to Euler regions. - : no data (no harmonic). 
fundamental. In order to sum-up, the one-way acoustic coupling (NS $\rightarrow$ Euler) presents good features in terms of energy conservation, even in most unfavourable cases.

\subsection{Standing wave in cavity}

A standing wave inside a cavity is simulated in order to assess the effectiveness of the two-way coupling. The cavity is composed of a NS sub-domain for $0<z^{*}<z_{\text {int }}^{*}$ and a Euler sub-domain for $z_{\text {int }}^{*}<z^{*}<1$ as depicted in figure 9. The second longitudinal mode $n=2$ is excited thanks to velocity fluctuations prescribed at $z^{*}=0$, with a SPL $=114 \mathrm{~dB}$ and a frequency $f_{s}=n c_{0} / 2 L=250 \mathrm{~Hz}$. This boundary is also set as non-reflective to avoid energy build up in the domain. The wavelength $\lambda_{s}$ is equal to the cavity length $L$. A purely reflective condition is set at $z^{*}=1$. In this way, the standing mode appears only if energy is properly sent back from the Euler to the NS sub-domain. The results are reported in figure 10 in terms of amplitude and phase of the standing wave, for a non tilted and a $33^{\circ}$ tilted interface. The coupled computations agree well with the analytical solution.

\subsection{Shock tube}

For space applications at high acoustic levels, shocked waves are expected to appear as already observed in the wave guide case. A shock tube case is finally simulated to validate the shock propagation and the shock-capturing scheme implemented in SPACE. NS and Euler sub-domains displayed in figure 11 are

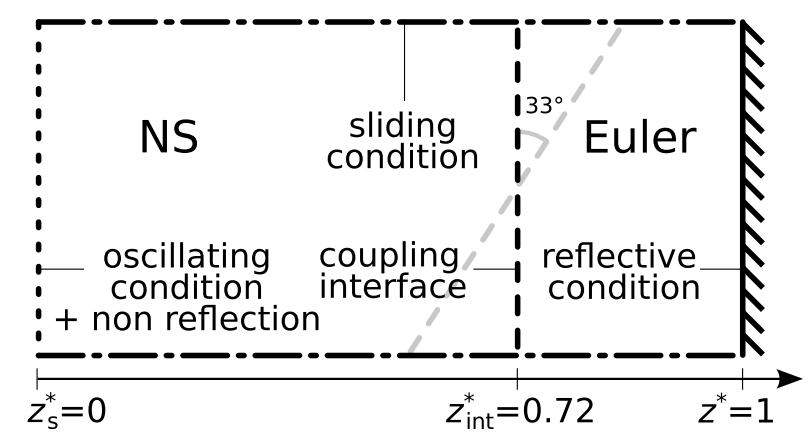

Fig. 9: sketch of the computational domain for the standing wave.

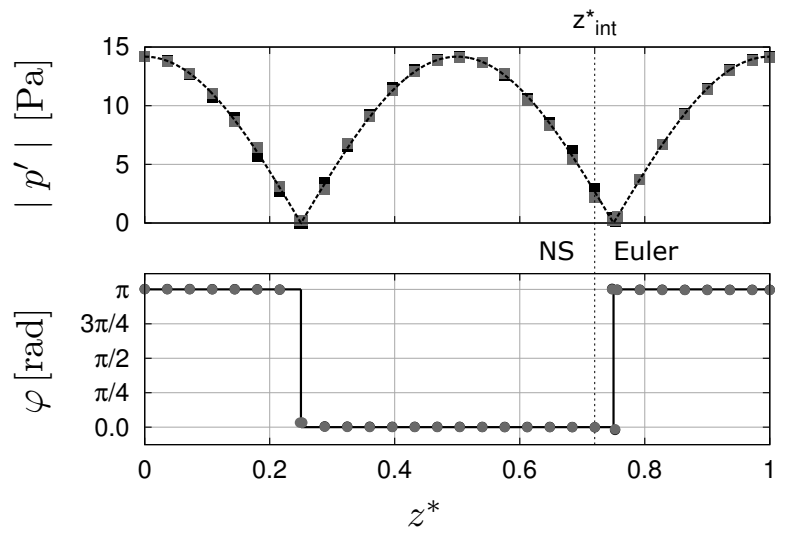

Fig. 10: amplitude and phase of the standing wave in the cavity for SPL $=114 \mathrm{~dB}$. -. : analytical amplitude; $\mathbf{\square} \mathbf{\square}$ : simulated amplitude with tilted $0 / 33^{\circ}$ interface; $\mathbf{Z}$ : analytical phase; $\bullet / \bullet:$ simulated phase with tilted $0 / 33^{\circ}$ interface. The coupling interface is located at $z_{\text {int }}^{*}$. 


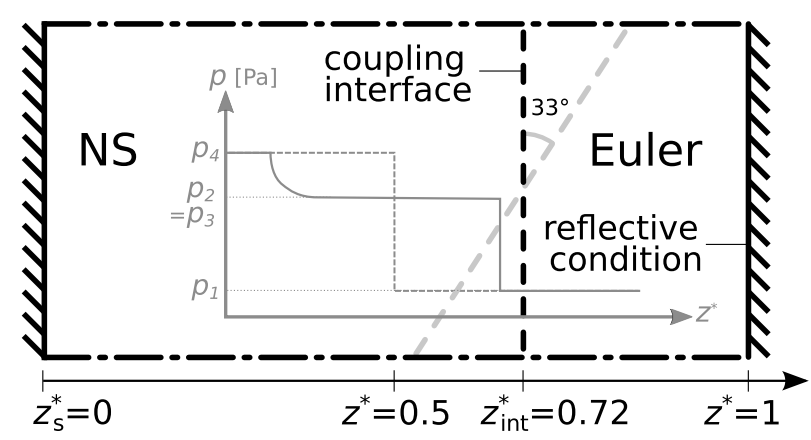

Fig. 11: sketch of the computational domain for the shock tube.

initialized at $p_{1}=100000 \mathrm{~Pa}$ for $z^{*}<0.5$ and $p_{4}=110000 \mathrm{~Pa}$ for $z^{*}>0.5$. A shock is thus created at the discontinuity and propagates in the increasing $z^{*}$ direction before being reflected in $z^{*}=1$, then in $z^{*}=0$.

Preliminary computations have shown that the shock-capturing scheme greatly improves the shock propagation in the Euler region by suppressing most of the spurious Gibbs oscillations with a less steep discontinuity as counterpart. The pressure, density and temperature ratios through the shock and the shock velocity are also conserved. A good agreement is found with the theoretical values obtained from Rankine-Hugoniot jump relations [50] as reported in table 2. Subscripts 1 and 2 are respectively associated with conditions ahead and behind the shock. A first approximation of the expected shock velocity [50] is given by

$$
c_{\text {shock }}=c_{1} \sqrt{\left(\frac{\gamma+1}{2 \gamma}\right)\left(\frac{p_{2}}{p_{1}}-1\right)+1} \simeq 354.4 \mathrm{~m} / \mathrm{s}
$$

where $c_{1}$ is the speed of sound in the gas ahead the shock. Numerical results are close to the theory within $1 \%$ error. The uncertainty is found to be larger with the use of the shock-capturing scheme because of the smoother discontinuity, as expected.

The shock is then reflected several times inside the tube. The shock profile after one, two, four and six round-trips is drawn in figure 12. The discontinuity is slightly smoothed after six round-trips but remains at the right location with respect to the analytical solution. Spurious oscillations can be noticed in the vicinity of the shock, but since the direct simulation agrees with the NS-Euler coupling (non tilted interface), it can not be attributed to the coupling procedure. On the other hand, stronger spurious oscillations are induced by a tilted interface but they are not amplified and the solution remains consistent.

\begin{tabular}{ccccc}
\cline { 2 - 5 } & $p_{2} / p_{1}$ & $\rho_{2} / \rho_{1}$ & $T_{2} / T_{1}$ & $c_{\text {shock }}[\mathrm{m} / \mathrm{s}]$ \\
\hline theoretical & 1.0486 & 1.0345 & 1.0137 & 354.4 \\
without shock capturing & 1.0488 & 1.0346 & 1.0137 & $351.8 \pm 0.8$ \\
with shock capturing & 1.0488 & 1.0346 & 1.0137 & $353.3 \pm 4.8$ \\
\hline
\end{tabular}

Table 2: pressure, density and temperature ratios through the shock and shock propagation velocity, see equation (7). 


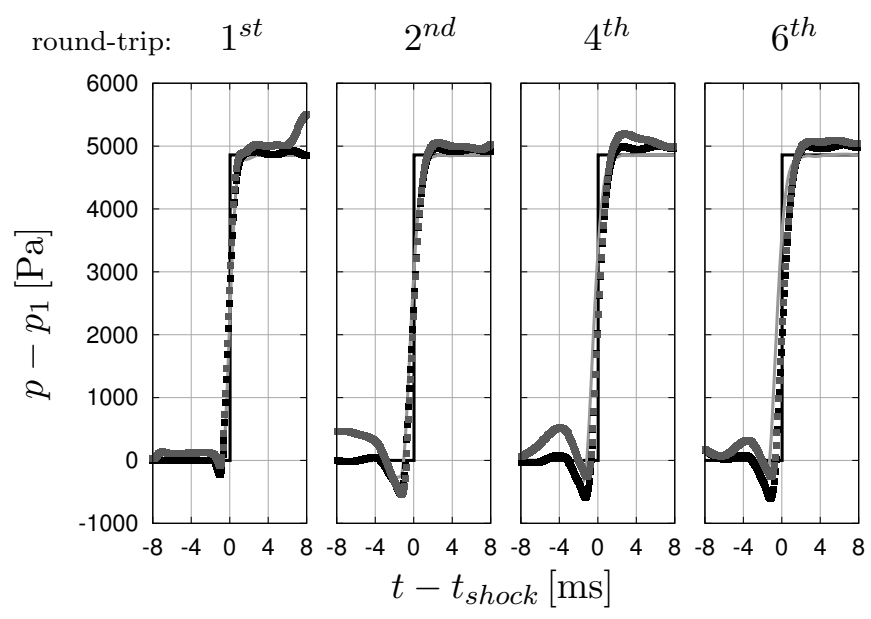

Fig. 12: shock wave propagation and multiple reflection case. - : analytical solution; $=$ : direct simulation; $\mathbf{\square}:$ NS-Euler coupling with non tilted interface; $\square$ : NS-Euler coupling with $33^{\circ}$ tilted interface.

\subsection{Convected isentropic vortex}

As mentioned in $\S 2.3$, vortices can intermittently cross the coupling interface. In order to quantify possible spurious noise induced by such events, a 2D convected isentropic vortex academic case is performed. The formulation proposed by Yee et al. [37] has been chosen. Although this problem is an exact solution of the full Euler equation, it is presently treated with the NS equations in the flow solver knowing that viscous effects are negligible as expected in practice. The vortex flow is defined as follow [29]:

$$
\left\{\begin{array}{l}
u_{z}=+\Gamma \frac{c_{0}}{2 \pi}\left(\frac{y-y_{0}}{r_{0}}\right) \exp \left(\frac{1-r^{2}}{2}\right)+u_{0} \\
u_{y}=-\Gamma \frac{c_{0}}{2 \pi}\left(\frac{z-z_{0}}{r_{0}}\right) \exp \left(\frac{1-r^{2}}{2}\right) \\
T=T_{0}\left(1-\Gamma^{2} \frac{(\gamma-1)}{8 \pi^{2}} \exp \left(1-r^{2}\right)\right)
\end{array}\right.
$$

with

$$
r^{2}=\left(\frac{z-z_{0}}{r_{0}}\right)^{2}+\left(\frac{y-y_{0}}{r_{0}}\right)^{2}
$$

where $z_{0}=0.5 \mathrm{~m}, y_{0}=0.5 \mathrm{~m}, r_{0}=0.1 \mathrm{~m}, T_{0}=298.15 \mathrm{~K}, c_{0}=\sqrt{\gamma r T_{0}}, u_{0}=100 \mathrm{~m} / \mathrm{s}$ and $\Gamma$ is the dimensionless vortex strength. The assumption of an isentropic disturbance leads to the relations

$$
\left\{\begin{array}{l}
\rho=\rho_{0}\left(1-\Gamma^{2} \frac{(\gamma-1)}{8 \pi^{2}} \exp \left(1-r^{2}\right)\right)^{1 /(\gamma-1)} \\
p=p_{0}\left(1-\Gamma^{2} \frac{(\gamma-1)}{8 \pi^{2}} \exp \left(1-r^{2}\right)\right)^{\gamma /(\gamma-1)}
\end{array}\right.
$$

It yields to a vortex initially centered at location $(z, y)=(0.5,0.5)$ and convected at $M=0.3$ in increasing $z$ direction as depicted in figure 13. The computational domain is composed of two NS sub-domains coupled on each side of the Euler sub-domain which allows to study the two-way crossing of vortical structures. Periodic boundary conditions are implemented for the non coupled sides of the NS sub-domains. Two 


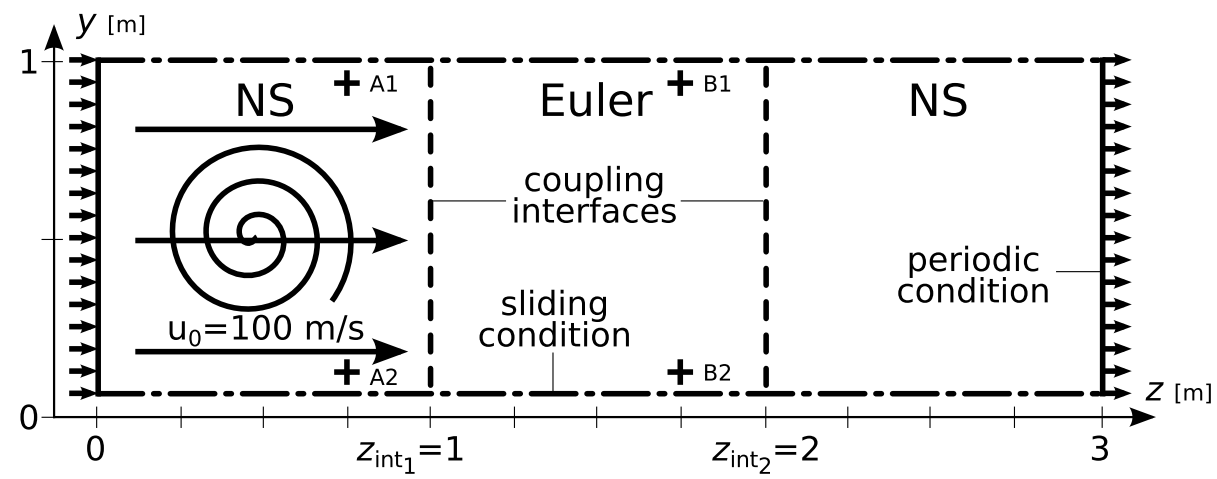

Fig. 13: sketch of the computational domain for the convected isentropic vortex. $\rightarrow$ : mean flow; + : probe locations.

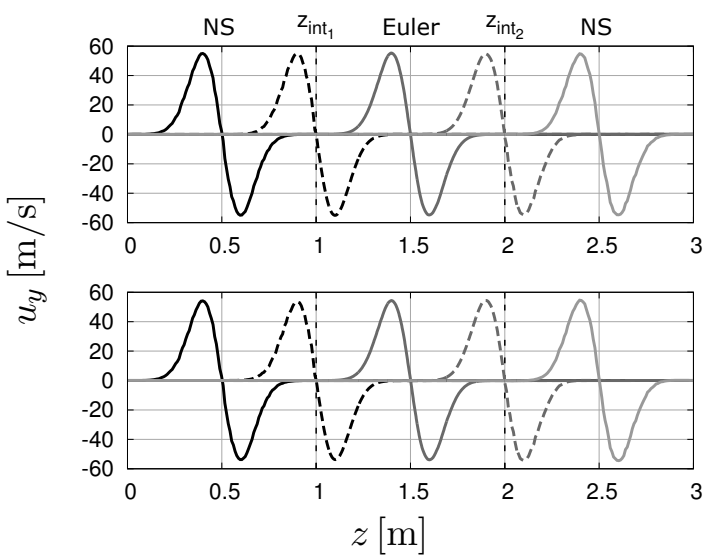

(a)

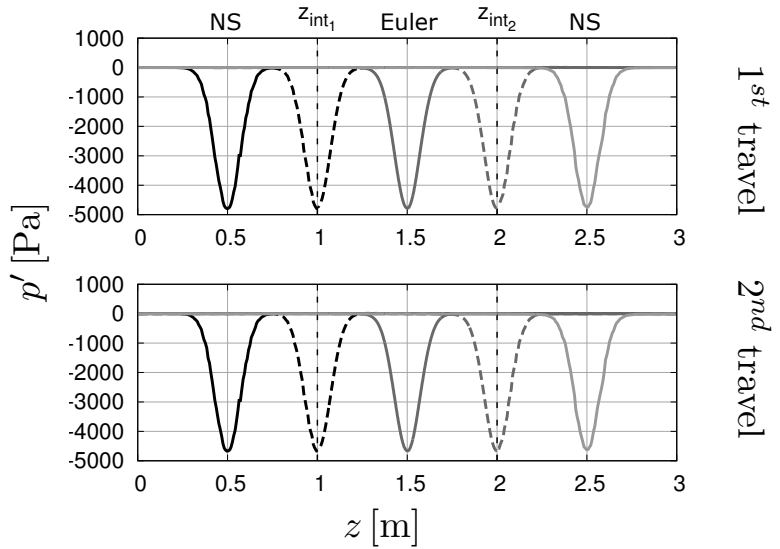

(b)

Fig. 14: (a) velocity and (b) pressure profile of the convected isentropic vortex at $-t=0[\mathrm{~ms}]$;

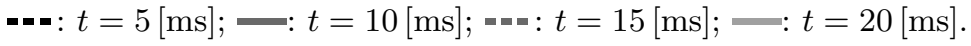

different vortex strengths are simulated, $\Gamma=1$ and $\Gamma=0.5$, leading to a pressure drop $|\Delta p|=4800 \mathrm{~Pa}$ and $|\Delta p|=1215 \mathrm{~Pa}$ at the vortex center.

Transversal velocity profiles and axial pressure profiles are respectively plotted in figure 14(a) and 14(b) for the first and the second travel (after one period) at five instants in the case $\Gamma=1$. No distortion is observed for the vortex itself. Nevertheless, spurious noise generation is found to occur when the vortex crosses the interface in both NS $\rightarrow$ Euler and Euler $\rightarrow$ NS ways. Pressure time signals recorded at locations A1/A2 and B1/B2 are respectively plotted in figure 15(a) and 15(b). These locations roughly correspond to the spurious noise peak directivities and it should be noted that, according to expression (10), pressure fluctuations directly associated with the vortex convection are negligible. Spurious noise is observed in both $\Gamma=1$ and $\Gamma=0.5$ cases. However, the amplitude of this noise remains weak with respect to the aerodynamic perturbation, i.e. about $0.2 \%$ for the $\Gamma=1$ case. In other words, based on the previously simulated supersonic free jet case [15], residual eddies which cross the interface were quite weak, typically $|\Delta p|<500 \mathrm{~Pa}$, resulting in an estimated spurious noise lesser than $100 \mathrm{~dB}$ while the actual noise in this area was greater than $140 \mathrm{~dB}$. Consequently, the signal-to-noise ratio is found to be rather reasonable. 


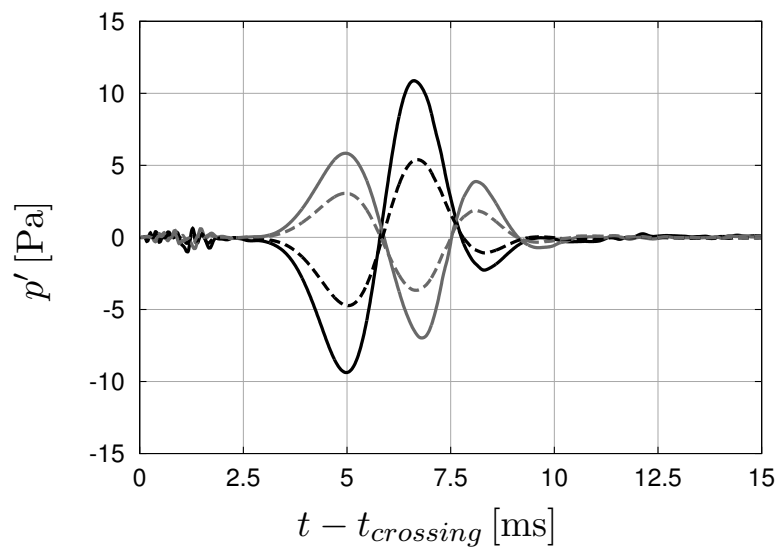

(a)

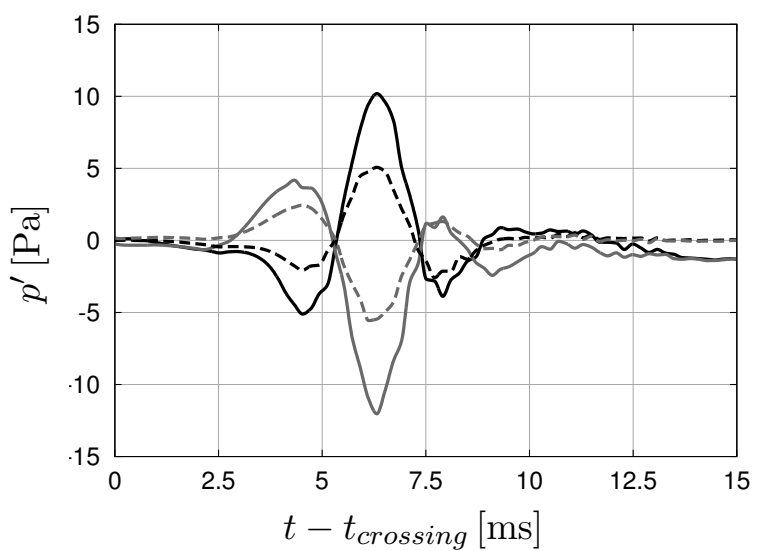

(b)

Fig. 15: spurious pressure wave generated when the vortex crosses the interface (a) NS $\rightarrow$ Euler at $z_{\text {int }}$, (b) Euler $\rightarrow \mathrm{NS}$ at $z_{i n t_{2}}$. $:$ A1 and B1 with $\Gamma=1 ;-\mathbf{- .}:$ A1 and B1 with $\Gamma=0.5$; —: A2 and B2 with $\Gamma=1 ; \cdots=$ A 2 and B2 with $\Gamma=0.5$.

\section{Application to a launch pad model}

\subsection{Geometry and parameters}

A three-dimensional launch pad model (LPM) is finally studied to assess the robustness and reliability of the coupling methodology with order mapping (see §2.4) in a more realistic configuration. The computational domain consists of a prismatic NS subdomain defined in range $-1.25 D_{j} \leq x \leq 1.25 D_{j}$, $0 \leq y \leq 2.5 D_{j}$ and $0 \leq z \leq k D_{j}$ where $k$ is variable and $D_{j}$ is the nozzle exit diameter. The Euler subdomain forms a simplified launch pad as displayed in figure 16, consisting of a cylindrical rocket motor body, a square duct with inner dimensions $2.5 \times 2.375 \times(12.5-k) D_{j}^{3}$ and a free field zone. An aperture on the top surface of the duct, so-called duct hole, has been introduced to model a possible acoustic device for noise reduction. The NS subdomain and the duct are closed by walls at $x=-1.25 D_{j}$ and $x=1.25 D_{j}$. The boundary going along the rocket body is set as a wall to model the launch umbilical tower. Non-reflective boundary conditions are imposed everywhere else. The whole domain is initially air at rest with $T_{0}=300 \mathrm{~K}$ and $p_{0}=101325 \mathrm{~Pa}$. Air is injected in the NS subdomain with a velocity $v_{0}=50 \mathrm{~m} / \mathrm{s}(M=0.14)$ in direction $-y$ through an inlet of diameter $D_{j}=0.4 \mathrm{~m}$ modeling the nozzle exit. An acoustic component corresponding to a $\mathrm{SPL}=160 \mathrm{~dB}$ is added to this mean flow through velocity fluctuations $\left(\left\|v^{\prime}\right\|=7 \mathrm{~m} / \mathrm{s}\right)$ oscillating at the fundamental frequency $f_{0}=500 \mathrm{~Hz}\left(\lambda_{0}=0.7 \mathrm{~m}=1.75 \mathrm{D}_{\mathrm{j}}\right)$. The resulting jet impacts the deflector in the NS subdomain, then exhausts in the duct through the coupling interface. Such a simplified space application involving a modeled acoustic source has also been performed by Harris et al. [28] in order to assess a one-way NS-Euler coupling based on unstructured meshes.

Several cases have been studied by moving the vertical coupling interface in the duct i.e. by varying the parameter $k$ as drawn in figure 16, plus an additional reference direct simulation (full NS) for comparison purposes. The mesh parameters, cut-off frequencies and computational costs are reported in table 3 .

\subsection{Computational cost}

The costs are estimated on a same number of processors and normalized by that of the direct simulation. As expected, the NS-Euler method with order mapping allows to dramatically decrease the number of required cells and the induced cost compared to the direct simulation at a same cut-off frequency. A structured finite-difference solver or an integral method would also have provided a low cost solution but these approaches are difficult to apply to realistic geometry. The LPM1 case is half as costly as the LPM3 one, suggesting that limit the NS sub-domain only to dominant acoustic generation regions could really be beneficial. 


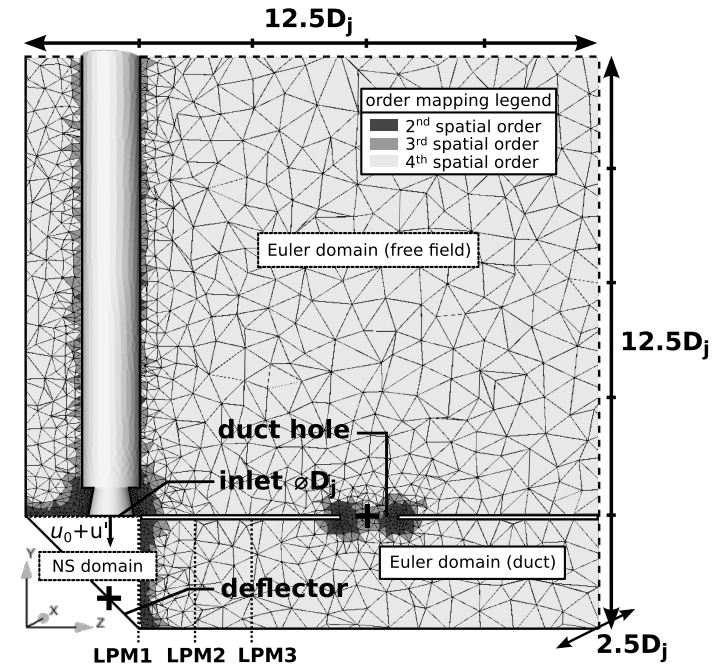

Fig. 16: computational domain and order mapping in the Euler sub-domain. — : wall; --- : non-reflective condition; $\cdot \cdots \cdot$ : coupling interface (3 cases); + : probe.

\begin{tabular}{cccccc}
\hline \multirow{2}{*}{ case } & \multirow{2}{*}{$k / D_{j}$} & \multicolumn{2}{c}{ cells $\times 10^{-6}$} & \multirow{2}{*}{$f_{c}[\mathrm{~Hz}]$} & normalized cost \\
\cline { 3 - 4 } & & $\mathrm{NS}$ & Euler & & \\
\hline LPM0 & - & 8.74 & - & 850 & $100 \%$ \\
LPM1 & 2.5 & 0.28 & 0.10 & 850 & $4.5 \%$ \\
LPM2 & 3.75 & 0.46 & 0.10 & 850 & $7.3 \%$ \\
LPM3 & 5.0 & 0.63 & 0.10 & 850 & $10 \%$ \\
\hline
\end{tabular}

Table 3: parameters and cost of the four launch pad model cases.

\subsection{Velocity and pressure field}

The goal of this short analysis is mainly to assess the robustness of the coupling methodology. In particular, the effect of the coupling interface location on the flow and acoustic fields is examined. A snapshot of the velocity norm superimposed with pressure iso-lines are presented in figure 17 in the plane $x=0$ for the LPM1 case. A deflected primary flow and multiple secondary flows near the nozzle and the hole regions can be noticed. It should be mentioned that before reaching the deflector, the primary flow generated a vortex ring which is intercepted by the vertical coupling interface only in the LPM1 case. The associated pressure drop at the interface is roughly $|\Delta p|=2000 \mathrm{~Pa}$. According to the discussion in section 3.6, the estimated generated spurious noise $(<110 \mathrm{~dB})$ is negligible compared to the actual acoustic source $(>150 \mathrm{~dB})$. Regarding the acoustic field, waves generated at the inlet are reflected and diffracted when impacting the deflector. The fundamental wavelength of the source $\lambda_{s}$ is smaller than duct transverse dimensions which induces a multi-modal acoustic propagation in the duct. Waves emitted in the free field arise from two main zones: the duct hole and the vicinity of the inlet. Since the duct hole diameter is of the order of the fundamental wavelength, a clear diffraction pattern can be observed. These waves interfere with those directly coming from the inlet region, forming interference patterns. In particular, a silent zone appears close to the top of the motor body as it can be seen on the root mean square pressure $p_{r m s}$ field further discussed in figure 19(a). Finally, no discontinuity is observed at the coupling interface, which demonstrates the good behaviour of the present methodology, even if a strong deflected flow crosses the interface in addition to acoustic fluctuations. 

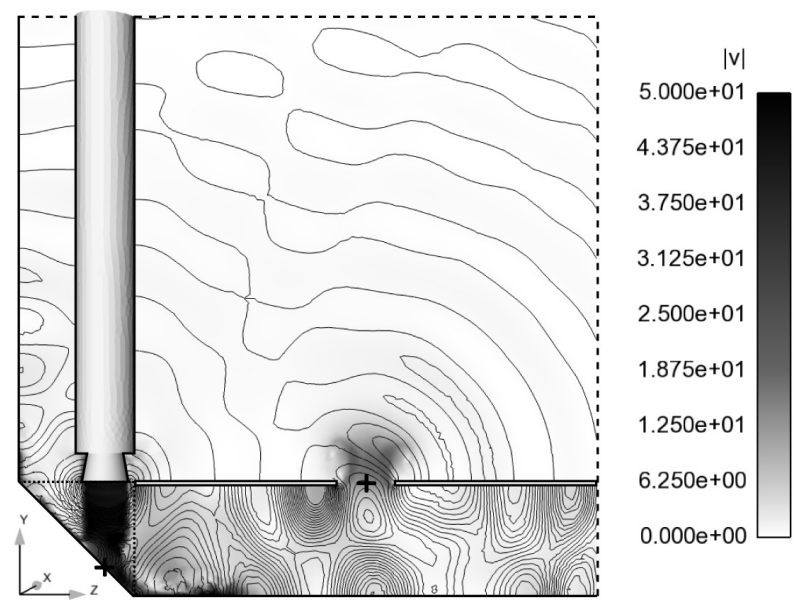

Fig. 17: snapshot of the velocity field in the LPM1 case at $t=0.07 \mathrm{~s}$ in the plane $x=0$, superimposed with

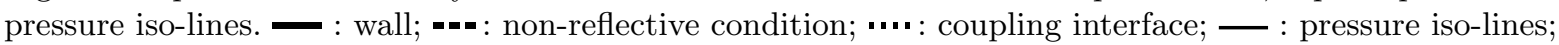
+ : probe.

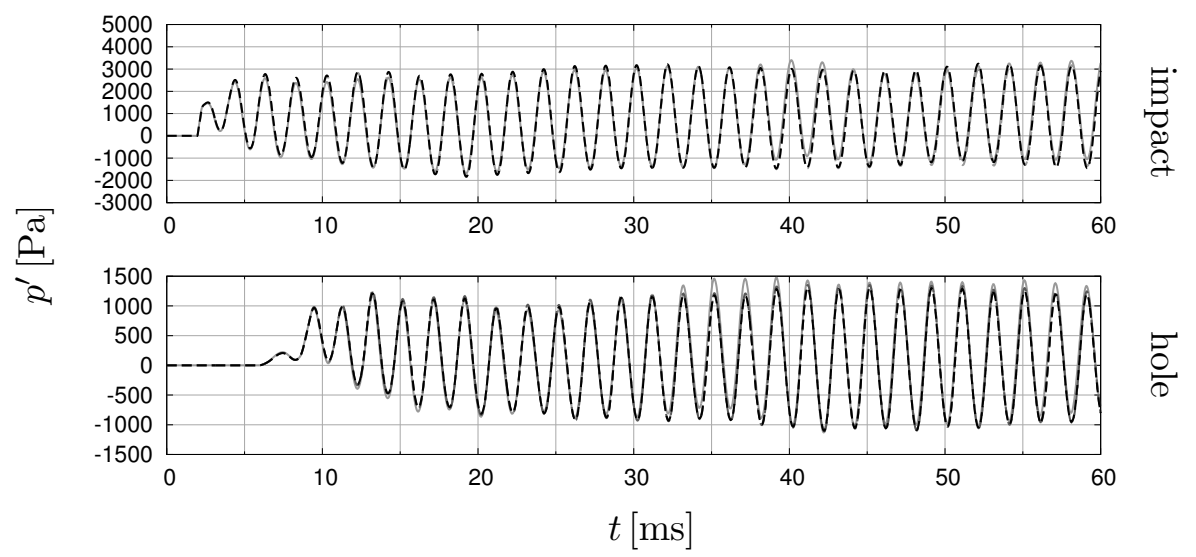

Fig. 18: pressure time signal recorded at probe locations as spotted in figure 16. - : direct simulation

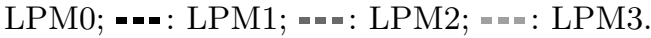

The transient pressure time signal recorded at two locations (i.e. at the point of impact in the NS domain and at hole center in the Euler domain, see + symbols in figure 16) are plotted in figure 18 for the direct simulation and the three coupling cases. The source main frequency is clearly visible and lower frequency transient deviations are also noticeable. The coupling interface intercepts the initial vortex ring and the deflected flow in the LPM1 case while a weak mean flow is only involved in the LPM3 case. A good agreement is found between all cases, proving the reliability of the present approach and suggesting that the coupling interface location has a minor influence if acoustic sources are restricted to the NS subdomain, even when vortical structures or a shear flow cross the interface. The LPM1 case shows a strong primary flow through the interface as depicted in figure 17 while the same flow does not have enough time to reach the interface in the LPM3 case. The pressure signal at the point of impact does not seem to be affected. Moreover, the induced spurious noise highlighted in the isentropic vortex case seems negligible as concluded previously. These results are corroborated by the comparison of the $p_{r m s}$ fields in figure 19 . Only very slight discrepancies are noticeable between the three cases LPM1, LPM2 and LPM3. 


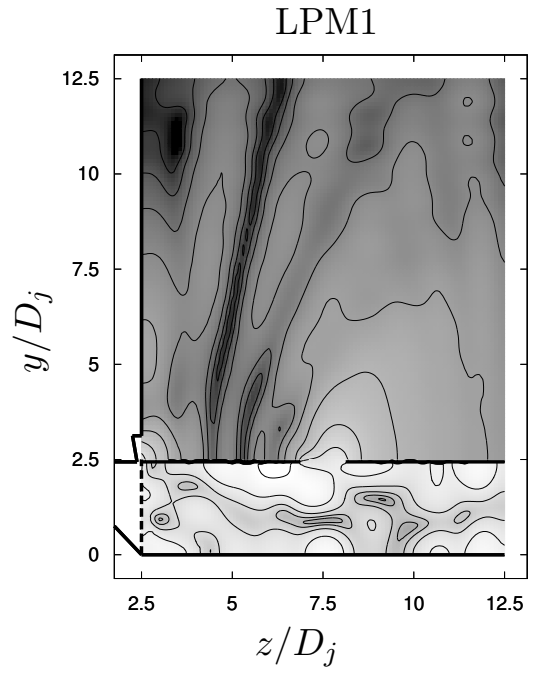

(a)

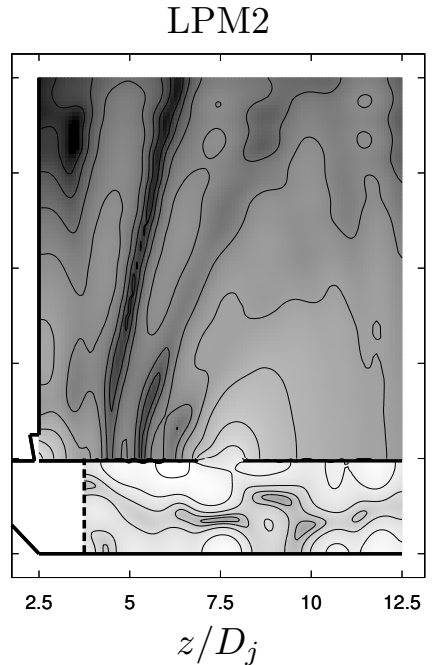

(b)

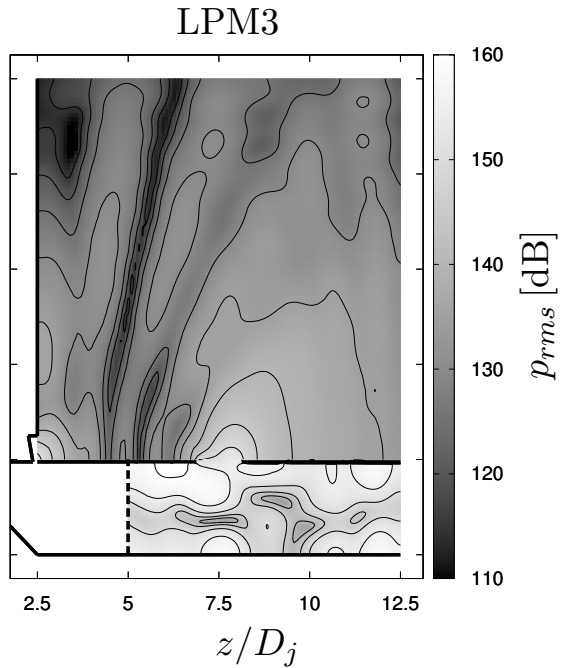

(c)

Fig. 19: root mean square pressure $p_{r m s}$ field in the plane $x=0$ with coupling interface (a) non offset, (b) offset by $1.25 D_{j}$, (c) offset by $2.5 D_{j}$. - wall; - - : coupling interface; —: $p_{r m s}$ iso-lines every $5 \mathrm{~dB}$.

\section{Conclusion}

A numerical methodology consisting in a NS-Euler two-way coupling on unstructured grids is assessed in this study. A flow solver has been coupled with a DG acoustic Euler solver, allowing to take into account installation and acoustic nonlinear effects. The solvers have been quickly presented. The coupling procedure and the use of high-order elements in the Euler region have been detailed. Several academic cases have been defined and performed to validate this approach. Its ability to accurately simulate both linear and nonlinear one-way acoustic phenomena has thus been demonstrated thanks to the pulsating sphere and the wave guide cases. The effectiveness of the two-way method have been checked on a standing cavity mode, a closed shock tube and a convected isentropic vortex. Consistent results have been found in terms of energy conservation, shock propagation and vortical structure convection through the interface. Spurious noise generated at the interface is noticeable in case of a non normal interface regarding the wave propagation direction and when vortices cross the interface. The level however remains sufficiently low to perform accurate aeroacoustic simulations. A launch pad model configuration has finally been considered, illustrating that the coupling can be easily applied to a complex geometry without inducing a prohibitive computational cost. Given the present results and the results of previous studies $[13,15,21]$, the methodology seems mature enough to be applied to a configuration involving a hot supersonic jet and a realistic launch pad, which will be carried out in further studies for noise prediction at launcher lift-off conditions.

Acknowledgements This study is supported by the french national space agency CNES and the ONERA's scientific direction. The authors are grateful to H. Lambaré, technical referee at CNES for launchers acoustic environment. Special thanks go to J. Troyes from ONERA for his technical support during all this work.

\section{Compliance with Ethical Standards}

Conflict of interests The authors declare that they have no conflict of interest. 


\section{References}

1. J.M. Seiner, in $9^{\text {th }}$ AIAA/NASA Aeroacoustics Conference (Williamsburg, VA, USA, 1984), 84-2275. doi:10.2514/6.1984-2275

2. C.K.W. Tam, Annual Review of Fluid Mechanics 27, 17 (1995). doi:10.1146/annurev.fl.27.010195.000313

3. C. Bailly, K. Fujii, Bulletin of the JSME 3(1, 15-00496), 1 (2016). doi:10.1299/mer.15-00496

4. B. Chemoul, E. Louaas, P. Roux, D. Schmitt, M. Pourcher, Acta Astronautica 48(5-12), 275 (2001). doi:10.1016/S0094-5765(01)00026-1

5. C.K.W. Tam, K. Viswanathan, K.K. Ahuja, J. Panda, Journal of Fluid Mechanics 615, 253 (2008). doi:10.1017/S0022112008003704

6. D. Gély, G. Elias, C. Bresson, H. Foulon, S. Radulovic, in $6^{\text {th }}$ AIAA/CEAS Aeroacoustics Conference (Lahaina, HI, USA, 2000), 2000-2026. doi:10.2514/6.2000-2026

7. K.M. Eldred, Acoustic loads generated by the propulsion system. SP 8072, NASA (1971)

8. J. Varnier, V. Koudriavstsev, A. Safronov, AIAA Journal 44(7), 1690 (2006). doi:10.2514/1.5087

9. M. Kandula, Journal of Sound and Vibration 309(3-5), 852 (2007). doi:10.1016/j.jsv.2007.06.078

10. J. Haynes, J. Kenny, in $15^{t h}$ AIAA/CEAS Aeroacoustics Conference (Miami, FL, USA, 2009), 2009-3160. doi:10.2514/6.2009-3160

11. N. de Cacqueray, C. Bogey, C. Bailly, AIAA Journal 49(10), 2171 (2011). doi:10.2514/1.J050952

12. J.B. Dargaud, J. Troyes, J.M. Lamet, L. Tessé, F. Vuillot, C. Bailly, Journal of Propulsion and Power 30(1), 164 (2014). doi:10.2514/1.B34824

13. J. Troyes, F. Vuillot, H. Lambaré, A. Espinosa Ramos, in 22 $2^{\text {nd }}$ AIAA/CEAS Aeroacoustics Conference (Lyon, France, 2016), 2016-2734. doi:10.2514/6.2016-2734

14. G. Brès, F. Ham, J. Nichols, S. Lele, AIAA Journal 55, 1164 (2017). doi:10.2514/1.J055084

15. A. Langenais, F. Vuillot, J. Troyes, C. Bailly, in $23^{r d}$ AIAA/CEAS Aeroacoustics Conference (Denver, CO, USA, 2017), 2017-3212. doi:10.2514/6.2017-3212

16. K. Fujii, T. Nonomura, S. Tsutsumi, International Journal for Numerical Methods in Fluids 64, 1412 (2010). doi:10.1002/fld. 2446

17. S. Tsutsumi, S. Ishii, K. Ui, S. Tokudome, K. Wada, in $53^{\text {rd }}$ AIAA Aerospace Sciences Meeting (Kissimmee, FL, USA, 2015), 2015-1007. doi:10.2514/6.2015-1007

18. A.S. Lyrintzis, International Journal of Aeroacoustics 2(2), 95 (2003). doi:10.1260/147547203322775498

19. A. Uzun, A.S. Lyrintzis, G.A. Blaisdell, International Journal of Aeroacoustics 3(4), 297 (2005). doi:10.1260/1475472043499290

20. G. Rahier, J. Prieur, F. Vuillot, N. Lupoglazoff, A. Biancherin, Aerospace Science and Technology 8, 453 (2004). doi:10.1016/j.ast.2004.04.005

21. J. Troyes, F. Vuillot, H. Lambaré, A. Espinosa Ramos, in $30^{\text {th }}$ International Symposium on Space Technology and Science (Kobe-Hyogo, Japan, 2015), 2015-399

22. N. de Cacqueray, C. Bogey, International Journal of Aeroacoustics 13(7 \& 8), 607 (2014). doi:10.1260/1475-472X.13.78.607

23. J. Utzmann, C.D. Munz, M. Dumbser, E. Sonnendrcker, S. Salmon, S. Jund, E. Frénod, Numerical Simulation of Turbulent Flows and Noise Generation (Springer, 2009), vol. 104, chap. Fluid-Acoustic Coupling and Wave Propagation, pp. 47-74. doi:10.1007/978-3-540-89956-3_3

24. R. Guenanff, Couplage instationnaire Navier-Stokes/Euler pour la génération et le rayonnement des sources de bruit aérodynamique. Ph.D. thesis, Université de Rennes I (2004)

25. G. Djambazov, C.H. Lai, K. Pericleous, Computing and Visualization in Science $3, \quad 9 \quad(2000)$. doi:10.1007/s007910050045

26. C. Bogey, S. Barré, D. Juvé, C. Bailly, Physics of Fluids 21, 1 (2009). doi:10.1063/1.3081561

27. A. Sescu, V. Sassanis, E. Collins, R. Harris, E. Luke, in $21^{\text {st }}$ AIAA/CEAS Aeroacoustics Conference (Dallas, TX, USA, 2015), 2015-2381. doi:10.2514/6.2015-2381

28. R. Harris, E. Collins, E. Luke, A. Sescu, in 21 $1^{\text {st }}$ AIAA/CEAS Aeroacoustics Conference (Dallas, TX, USA, 2015), 2015-2538. doi:10.2514/6.2015-2538

29. O. Labbé, C. Peyret, G. Rahier, M. Huet, Computers \& Fluids 86, 1 (2013). doi:10.1016/j.compfluid.2013.07.013

30. M. Borrel, L. Halpern, J. Ryan, in 20 th AIAA Computational Fluid Dynamics Conference (Honolulu, HI, USA, 2011), 2011-3047. doi:10.2514/6.2011-3047

31. R. Léger, C. Peyret, S. Piperno, AIAA Journal 50(2), 338 (2012). doi:10.2514/1.J051110

32. O. Labbé, C. Peyret, in $6^{\text {th }}$ International Conference on Computational Methods for Coupled Problems in Science and Engineering (Venice, Italy., 2015), pp. 501-511

33. J. Hardin, J. Ristorcelli, C. Tam (eds.). ICASE/LaRC Workshop on Benchmark Problems in Computational Aeroacoustics (CAA), NASA/CP-3300 (1994)

34. C. Tam, J. Hardin (eds.). Second Computational Aeroacoustics (CAA) Workshop on Benchmark Problems, NASA/CP-3352 (1997)

35. J. Hardin, D. Huff, C. Tam (eds.). Third Computational Aeroacoustics (CAA) Workshop on Benchmark Problems, NASA/CP-2000-209790 (2000)

36. M. Dahl, E. Envia, D. Huff, C. Tam (eds.). Fourth Computational Aeroacoustics (CAA) Workshop on Benchmark Problems, NASA/CP-2004-212954 (2004)

37. H. Yee, N. Sandham, M. Djomehri, Journal of Computational Physics 150, 199 (1999). doi:10.1006/jcph.1998.6177

38. A. Refloch, B. Courbet, A. Murrone, P. Villedieu, C. Laurent, P. Gilbank, J. Troyes, L. Tessé, G. Chaineray, J.B. Dargaud, E. Quémerais, F. Vuillot, Aerospace Lab Journal 2(11), 1 (2011)

39. P. Delorme, P. Mazet, C. Peyret, Y. Ventribout, Comptes Rendus Mécanique 333(9), 676 (2005). doi:10.1016/j.crme.2005.07.007

40. A. Langenais, J. Troyes, C. Peyret, G. Chaineray, in 13eme Congrès Franais d'Acoustique (Le Mans, France, 2016), 000150

41. C. Hirsch, Numerical Computation of Internal and External Flows, Volume 2: Computational Methods for Inviscid and Viscous Flows, 978-0-471-92452-4, vol. 2 (John Wiley \& Sons, 1990) 
42. R. Hartmann, P. Houston, Journal of Computational Physics 183, 508 (2002). doi:10.1006/jcph.2002.7206

43. C. Peyret, P. Delorme, in 13 $3^{\text {th }}$ AIAA/CEAS Aeroacoustics Conference (Rome, Italy, 2007 ), $2007-3475$. doi:10.2514/6.2007-3475

44. E. Quémerais. Coupling with interpolation parallel interface (2016). URL http://sites.onera.fr/cwipi/

45. G. Cunha, S. Redonnet, International Journal for Numerical Methods in Fluids 71(7), 910 (2012). doi:10.1002/fld.3693

46. C.K.W. Tam, AIAA Journal 33(10), 1788 (1995). doi:10.2514/3.12728

47. ISO 9613-1:1993 - acoustics - attenuation of sound during propagation outdoors - part 1: Air absorption (1993)

48. D.T. Blackstock, Journal of the Acoustical Society of America 39(6), 1019 (1966). doi:10.1121/1.1909986

49. J.A. Gallagher, D.K. McLaughlin, in $7^{t h}$ AIAA Aeroacoustics Conference (Palo Alto, CA, USA, 1981), 81-2041. doi:10.2514/6.1981-2041

50. H. Liepmann, A. Roshko, Element of Gasdynamics (John Wiley \& Sons, 1959), chap. One-dimensional wave motion. doi:10.1002/aic.690050234 